\title{
Can we evaluate the predictability of financial markets?
}

In the summer of 2007, the International Institute of Forecasters (IIF) asked us to organize a workshop on "Predictability of Financial Markets", which took place on the 16th and 17th of January, 2009, in the beautiful, historical building of the Institute of Economics and Management (ISEG) in Lisbon. Nine outstanding invited speakers presented papers in the area of time series analysis of financial data, which were then discussed by nine other experts. This special issue provides peerreviewed, corrected and updated versions of seven of these papers, with additional comments by the discussants. Sadly, the paper by the Nobel Laureate Sir Clive Granger could not be finished, as he passed away in May 2009. We will always have the memories of his talk, which has been commented on here by Antonio García-Ferrer. In addition, the paper by Stephen Taylor entitled "A multi-horizon comparison on density forecasts for the S\&P 500 index returns and option prices" was unfortunately not made available for this special issue.

The analysis of the predictability of financial markets is interesting from the point of view of both academics and practitioners. There have recently been two special issues of the International Journal of Forecasting which were closely related to this topic. In their introduction to the first of these issues, Clements, Franses, and Swanson (2004) argue that the relative poor predictive performance of non-linear models calls for substantial further research on special topics such as joint and conditional predictive density evaluation, loss functions, the estimation and specification of nonlinearities, and data-mining. The second relevant special issue, edited by Clements, Milas, and van Dijk (2009), deals with topics such as the usefulness of realized and implied volatilities, non-parametric estimators of daily volatilities, and the impact of long memory, level shifts or macroeconomic announcements on volatility modeling.

The papers included in the present special issue contribute to some of the areas which Clements et al. (2004, 2009) pointed out as interesting: (i) obtaining prediction intervals or even the whole density for future returns, taking into account the evolution of the volatility over time-see De Gooijer and Hyndman (2006) for a discussion of the interest of this topic; (ii) modeling some features of univariate volatilities, such as long memory, which are fundamental to many models of portfolio selection, risk management, and asset valuation; (iii) identifying multivariate models that use the dynamic correlations among different assets for predicting the volatilities of financial returns.

The present special issue includes two papers which are related to the prediction of quantiles and densities. The first is authored by Harvey and Oryshchenko, who propose the use of time-varying quantiles and copulas to represent nonlinear relationships between pairs of time series. They suggest a procedure for estimating timevarying distributions based on non-parametric kernel estimation and EWMA filters. After the distributions have been estimated, the quantiles can be obtained by inverting them. In her commentary on this paper, Pérez puts forward several questions about particular technical aspects of this procedure. In particular, she shows that the method is robust with respect to the choice of the different bandwidths needed to obtain the kernels and to the grid used to estimate the quantile.

The second paper related to the prediction of densities is authored by González-Rivera and Arroyo, who present new ideas related to modeling the evolution of intervals, or even the whole histogram for a given variable, instead of focusing on just one data point at a time. The paper is vividly discussed by João Nicolau, who points out various interesting areas for future research for histogram time series (HTS) and interval time series (ITS). In particular, Nicolau stresses the need to make specification, estimation and testing, integral components of the ITS and HTS research agenda. He also points out some shortcomings of HTS, as histograms do not provide direct measures of forecasting accuracy.

Finally, Carnero briefly reviews and discusses the ideas of the paper presented by Stephen Taylor at the workshop, which dealt with the comparison of multi-step forecasts of S\&P500 returns when they are constructed using option-based and historical forecasts. Carnero proposes alternative criteria for ranking density forecasts; in particular, she focuses on sharpness and wonders whether the main conclusions on the ranking of the density forecasts could be maintained when sharpness 
is used instead of the out-of-sample likelihood criteria implemented by Shackleton, Taylor, and Yu (2006). She also highlights the potential improvement in point and interval forecasts that can be obtained when constructing them from the density forecasts.

The univariate prediction of financial time series is the topic of two other papers published in this special issue. First, Rohit Deo discusses the biases encountered in the predictive regression for VAR models, and evaluates the advantages of a weighted least squares approximated restricted maximum likelihood estimator. Paulo Rodrigues discusses this paper and stresses the doors it opens for future research. In particular, he says that it will be interesting to see how this estimator will cope with empirically relevant features of the data, such as cointegration.

Second, the paper by Baillie, Kongcharoen, and Kapetanios discusses the potential of ARFIMA models for forecasting and compares the predictions obtained when the parameters are estimated using two alternative methods. Specifically, they consider the parametric Maximum Likelihood (ML) estimator and a two-step estimator based on a semiparametric Local Whittle (LW) estimator for the long memory parameter, followed by the estimation of the ARMA components of the fractionally differenced series. After carrying out several Monte Carlo experiments, they show that the two-step method has difficulties in estimating the long-memory parameter, relative to the ML estimator. Furthermore, they show that the predictor based on MLE is superior (in the MSE sense) to the predictor based on the two step LW estimation. The discussant, Josu Arteche, suggests that when there is misspecification of the short-memory dependency, the forecasts obtained when the parameters are estimated by semiparametric or local alternatives may have better properties than those based on fully parametric estimators.

There are three further papers related to the multivariate modeling and prediction of financial time series. First, Frank Diebold and Kamil Yilmaz discuss new measures of cross-markets volatility spillovers. This is a fundamental issue in describing financial markets, as it allows the volatility transmissions among different markets to be measured, an issue that has been proved to be very important nowadays in understanding what is happening in the global financial world. Raquel Gaspar discusses this paper and stresses the fact that the authors introduce the important concept of directional spillovers. She lists various important empirical studies that can be made using the author's methodology and that could potentially clarify some momentous practical issues.

Second, García-Ferrer, Gónzalez-Prieto, and Peña show how the novel idea of independent components can be used to extract information on the nonlinear components of multivariate time series by maximizing the kurtosis. They apply Independent Component Analysis (ICA) to conditionally heteroscedastic non-Gaussian stock returns in order to separate the independent sources of variation. The new model, called GICA-GARCH, avoids the curse of dimensionality by modeling each of the underlying independent components with a single univariate model. The discussant, Pilar Poncela, points out three aspects of this new methodology that deserve further research: (i) whether the ordering and extraction of the components should be carried out using the same criteria; (ii) how the number of common factors should be chosen; and (iii) the statistical properties of the common factors extracted.

Third, Viceira focuses on the interest in predictions from the financial point of view instead of the methodological point of view. He analyzes whether the bond risk, as measured by the CAPM beta, is related to yield spreads and the short term interest rate, and concludes that the yield spread can forecast the covariance between bonds and stocks and the bond beta. However, it does not forecast the bond volatility. The discussant, Andreas Heinen, suggests alternative methodologies for analyzing this relationship, pointing out several problems with using a regression model to represent the dependence of covariances and betas.

Finally, Clive Granger presented at the workshop a general overview of topics of interest in the analysis of financial time series. Among many other ideas, he mentioned that although volatility is fluctuation, not risk, it is important to model it because there is a new market where volatility is traded as a commodity. In terms of modeling, he pointed out the advantages of time-varying parameter models for approximating nonlinear dependencies and the importance of modeling and forecasting the shape of distributions and their quantiles. In this issue, we include a discussion of Granger's presentation by Antonio García-Ferrer.

\section{Acknowledgements}

We are very grateful to all participants at the workshop on "Predictability of Financial Markets". Financial support from FCT is acknowledged by the first author and from the Spanish Government project ECO2009-08100 is acknowledged by the second author. Obviously, any errors remain our responsibility.

\section{References}

Clements, M. P., Franses, P. H., \& Swanson, N. R. (2004). Forecasting economic and financial time-series with non-linear models. International Journal of Forecasting, 20(2), 169-183.

Clements, M. P., Milas, C., \& van Dijk, D. (2009). Introduction: forecasting returns and risk in financial markets using linear and nonlinear models. International Journal of Forecasting , 25(2), 215-217.

De Gooijer, J., \& Hyndman, R. (2006). 25 years of time series forecasting. International Journal of Forecasting, 22(3), 443-473.

Shackleton, M. B., Taylor, S. J., \& Yu, P. (2006). A multi-horizon comparison of density forecasts for the SEP 500 using index returns and option prices. Manuscript.

Nuno Crato

CEMAPRE and ISEG, Universidade Técnica de Lisboa, Portugal

Esther Ruiz*

Dpt. Estadística and Instituto Flores de Lemus, Universidad Carlos III de Madrid, Spain E-mail address: ortega@est-econ.uc3m.es.

* Corresponding editor. Tel.: +3491 6249851; fax: +34 91 6249849 . 